\title{
Niveles de Referencia para Diagnóstico en Procedimientos Radiológicos Dentales: Una Guía Práctica
}

\author{
Diagnostic Reference Levels in Dental Radiological Procedures: A Practical Guide
}

\author{
Carlos Ubeda'; Diego Nocetti'; Marco Aragón²; Gonzalo Aragón; Diego Aragón ${ }^{4}$ \& Oscar Medina ${ }^{5}$
}

UBEDA, C.; NOCETTI, D.; ARAGÓN, M.; ARAGÓN, G.; ARAGÓN, D. \& MEDINA, O. Niveles de referencia para diagnóstico en procedimientos radiológicos dentales: una guía práctica. Int. J. Odontostomat., 14(4):610-616, 2020.

RESUMEN: Las exposiciones médicas con radiación ionizante son actualmente la principal fuente de exposición a la radiación artificial a nivel global, entre ellas, los exámenes dentales corresponden a uno de los más frecuentes. Para prevenir dosis innecesariamente altas a los pacientes durante estas exposiciones, la Comisión Internacional de Protección Radiológica (ICRP) recomienda la utilización de Niveles de Referencia para Diagnóstico (DRLs), como una herramienta efectiva de ayuda a la optimización de la protección radiológica en la exposición médica de pacientes. Dado que la legislación de Chile aún no ha incorporado el uso de los DRLs, el presente trabajo de revisión tuvo como objetivo analizar la publicación № 135 de la ICRP para generar un documento que sirva de guía para los odontólogos, explicando de manera didáctica y en un lenguaje sencillo, pero técnico, los principales aspectos a tener en cuenta para establecer los DRLs en procedimientos de radiología dental.

PALABRAS CLAVE: niveles de referencia para diagnóstico, radiología dental, seguridad y protección radiológica, exposición médica.

\section{INTRODUCCIÓN}

El promedio anual de exposición a la radiación procedente de todas las fuentes entre la población

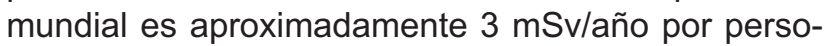
na. De esta exposición, el $80 \%(2,4 \mathrm{mSv})$ de la dosis anual que recibe una persona de todas las fuentes de radiación se debe al radón y a otras fuentes de radiación de origen natural (radiación natural de fondo), el $19,7 \%(0,6 \mathrm{mSv})$ se debe al uso de la radiación con fines médicos (incluidos los dentales) y el $0,3 \%$ restante (aproximadamente 0,01 $\mathrm{mSv}$ ) procede de otras fuentes de radiación creadas por el ser humano (Organización Mundial de la Salud, 2016).

Los procedimientos radiológicos dentales desempeñan un papel importante en el diagnóstico, tratamiento y seguimiento de las patologías que presentan los pacientes en sus piezas dentales o sistema masticatorio (Ruiz, 2005). El Comité Científico de las Naciones Unidas sobre los efectos de la Radiación Ató- mica clasificó la radiografía dental como uno de los procedimientos radiológicos más frecuentes (United Nations Scientific Committee on the Effects of Atomic Radiations, 2008). Según el modelo lineal sin umbral, el riesgo radiológico esperado para procedimientos de diagnóstico de baja dosis, es pequeño, pero mayor que cero. Los efectos en la salud asociados a la radiación son de naturaleza estocástica, como lo demuestra la mayor probabilidad de inducción carcinogénica al aumentar la dosis absorbida (International Commission on Radiation Protection, 2007a).

Se deben adoptar medidas de protección radiológica a fin de prevenir, en lo posible, dosis innecesariamente altas durante las exposiciones médicas dentales a los pacientes (Ubeda, 2018; Ubeda et al., 2018). De acuerdo a las recomendaciones de la Comisión Internacional de Protección Radiológica (ICRP) (International Commission on Radiation Protection,

\footnotetext{
${ }^{1}$ Departamento de Tecnología Médica, Facultad de Ciencias de la Salud, Universidad de Tarapacá, Arica, Chile.

${ }^{2}$ Ortodoncista. Clínica Arisa, Arica, Chile.

${ }^{3}$ Escuela de Medicina, Pontificia Universidad Católica de Chile.

${ }^{4}$ Escuela de Medicina, Universidad de Valparaíso, Valparaíso, Chile.

${ }^{5}$ Consultorio SAPUNAR, Dirección Municipal de Salud de Arica, Chile.
} 
2007a), no se recomiendan límites de dosis ni restricciones de dosis para pacientes individuales, porque los mismos pueden reducir la eficacia del diagnóstico, provocando más perjuicio que beneficio. Por consiguiente, el énfasis se debe poner en la justificación de los exámenes radiológicos, en la optimización de la protección y en la utilización de los Niveles de Referencia para Diagnóstico (DRLs).

Los DRLs han demostrado, por medio de diversos estudios, ser una herramienta efectiva que ayuda a la optimización de la protección radiológica en la exposición médica de pacientes para diagnóstico y procedimientos de intervencionismo (Hart et al., 2009, 2012). Sin embargo, su aplicación en Chile se ha visto seriamente limitada porque la legislación que regula el uso seguro de las exposiciones médicas con radiaciones ionizantes está desactualizada (Decreto Supremo $N^{\circ} 133,1984$; Decreto Supremo $\left.N^{\circ} 3,1985\right)$ respecto de las últimas recomendaciones internacionales del Organismo Internacional de Energía Atómica, el cual destaca la importancia de conocer y aplicar los DRLs (Organismo Internacional de Energía Atómica, 2016).

Así, el presente artículo de revisión narrativa tuvo como objetivo analizar profundamente la publicación N ${ }^{\circ} 135$ de la ICRP (International Commission on Radiation Protection, 2017) para generar un documento que sirva como guía práctica para los odontólogos, explicando de manera didáctica y en un lenguaje sencillo, pero técnico, los principales aspectos a tener en cuenta para establecer los DRLs en procedimientos de radiología dental.

\section{DESARROLLO}

Antecedentes. En el año 1990 fue mencionado por primera vez el concepto de DRL (International Commission on Radiation Protection, 1990), donde se indicaba que: "Se debería considerar el uso de restricciones de dosis, o niveles de investigación, seleccionados por la autoridad profesional o reguladora apropiada, para su aplicación en los procedimientos diagnósticos comunes. Estas restricciones se deberían aplicar con cierta flexibilidad, con el fin de permitir el uso de ciertas dosis cuando así lo indique una fundada valoración clínica". Su uso, finalmente fue recomendado en la Publicación 73 de la ICRP, en el año 1996 (International Commission on Radiation Protection, 1996) y se indicó que: "Los niveles de referencia se usan en el diagnóstico clínico para indicar si, en con- diciones de rutina, los niveles de dosis al paciente o la actividad administrada (la cantidad de material radiactivo) en un procedimiento de imagen específico, son excepcionalmente altos o bajos para ese procedimiento. En ese caso, debe comenzarse una revisión local para determinar si la protección se ha optimizado adecuadamente o si se necesita una acción correctora". En el año 2001 se publicaron algunas aclaraciones adicionales a través de una guía de apoyo de la ICRP (International Commission on Radiation Protection, 2001), no habiendo cambios sustanciales en los siguientes documentos de la Comisión 103: (International Commission on Radiation Protection, 2007a) y 105 (International Commission on Radiation Protection, 2007b) hasta el reporte 135 publicado en el año 2017 (International Commission on Radiation Protection, 2017).

Definiciones. La ICRP (International Commission on Radiation Protection, 2017) recomienda el uso de cuatro términos para comprender los DRLs:

a. DRL: Es una forma de nivel de investigación utilizada como herramienta para ayudar a la optimización de la protección en la exposición médica de pacientes sometidos a procedimientos de diagnóstico e intervencionismo.

b. Magnitud de DRL: Una magnitud que sea fácilmente medible y que permita evaluar la cantidad de radiación utilizada para realizar una tarea clínica concreta (Tablas I y II).

c. Valor de DRL: Un valor numérico arbitrario de una magnitud de DRL. Se habla de "valor típico de DRL" cuando se refiere a centros sanitarios que tengan una o varias salas de rayos $X$, o un pequeño grupo de instalaciones que utilicen una nueva técnica. En este caso se utiliza la mediana de la magnitud seleccionada. Además, se establece el percentil 75 de la distribución de las medianas de la magnitud DRL elegida en:

(a) unos pocos centros sanitarios, una localidad o una ciudad (denominado "valor local de DRL"); (b) múltiples instalaciones en todo el país (denominado "valor nacional de DRL"); o (c) múltiples países en una misma región del mundo (denominado "valor regional de DRL", utilizando el valor de la mediana de los valores nacionales disponibles).

d. Proceso DRL: Ciclo para el establecimiento de los valores de DRLs, usándolos como una herramienta para la optimización, y luego determinar los valores 
UBEDA, C.; NOCETTI, D.; ARAGóN, M.; ARAGÓN, G.; ARAGÓN, D. \& MEDINA, O. Niveles de referencia para diagnóstico en procedimientos radiológicos dentales: una guía práctica. Int. J. Odontostomat., 14(4):610-616, 2020.

actualizados de DRLs como herramientas para futuras optimizaciones.

\section{Consideraciones fundamentales para establecer} Ios DRLs. Los DRLs se definen para los diferentes tipos de equipos y exámenes (indicaciones clínicas) en grupos de pacientes de acuerdo a su rango de edad y fundamentalmente el peso. Sin embargo, la radiología dental y en particular las modalidades de radiografía intraoral y panorámica, son relativamente independientes del tamaño del paciente. Estos equipos dentales intraorales son de tensión (kilovoltaje) e intensidad de corriente (miliapere) fijos y poseen un temporizador ajustable. Por lo anterior, la mejor opción es la medición directa con detectores de radiación por un físico médico cuando se realizan las pruebas de control de calidad, en vez de hacer una medición en pacientes individuales (International Commission on Radiation Protection, 2017).

Reglamentación. Para una práctica adecuada se recomienda disponer de requisitos reglamentarios para establecer los valores de DRLs, su aplicación y la optimización de la protección en las exposiciones médicas dentales. En diferentes partes del mundo, la gestión de dosis a los pacientes se aborda de distintas maneras (Martin et al., 2013), de modo que se necesitará creatividad a la hora de establecer los DRLs e implementar los programas de optimización.

Selección de la magnitud y valor de DRL. La magnitud seleccionada deberá estar en directa relación con la modalidad estudiada (Tablas I y II). Por otra parte, los DRLs se pueden establecer en base a la distribución de las medianas para las magnitudes de DRLs medidas en una muestra de pacientes individuales o equipos (radiografía intraoral y panorámica) en áreas geográficas diferentes. La mediana se considera un estimador más robusto que la media, y con un mayor número de datos de dosis a pacien-
Tabla II. Magnitudes adecuadas para establecer niveles de referencia para diagnóstico (DRLs).

\begin{tabular}{lcc}
\hline Modalidad & $\begin{array}{c}\text { Magnitud } \\
\text { recomendada }\end{array}$ & $\begin{array}{c}\text { Unidad } \\
\text { recomendada }\end{array}$ \\
\hline Radiografía intraoral & $\mathrm{Ka}, \mathrm{i}$ & $\mathrm{mGy}$ \\
Radiografía & $\mathrm{PKA}$ & $\mathrm{mGy} \cdot \mathrm{cm}^{2}$ \\
panorámica & $\mathrm{DLP}$ & $\mathrm{mGy} \cdot \mathrm{cm}$ \\
Radiografía lateral & $\mathrm{Ka}, \mathrm{e}$ & $\mathrm{mGy}$ \\
de cráneo & $\mathrm{PKA}$ & $\mathrm{mGy} \cdot \mathrm{cm}^{2}$ \\
CBCT & $\mathrm{Ka}, \mathrm{r}$ & $\mathrm{mGy}$ \\
& $\mathrm{CTDlvol}$ & $\mathrm{mGy}$ \\
& $\mathrm{DLP}$ & $\mathrm{mGy} \cdot \mathrm{cm}^{2}$ \\
& $\mathrm{PKA}$ & $\mathrm{mGy} \cdot \mathrm{cm}^{2}$ \\
\hline
\end{tabular}

CBCT, Tomografía computada de haz cónico; Ka,e, kerma en aire en la superficie de entrada; PKA, producto kerma en aire área; Ka,i, kerma incidente en aire; Ka,r, kerma incidente en aire en el punto de referencia a la entrada del paciente; CTDIvol, índice de dosis en tomografía computarizada (volumen); DLP, producto dosis longitud. (Información extraída y adaptada por los autores desde la tabla 2.4 de la publicación ICRP-135 (International Commission on Radiation Protection, 2017)).

tes, se considera que proporciona una cantidad que es más representativa de la población de pacientes. Para las áreas en las que se puedan incluir 10-20 servicios, el DRL local se establecerá como el tercer cuartil de la distribución de las medianas. Ello puede ser útil para identificar las unidades de rayos $X$ donde se requiera más atención para la optimización. Para un número menor de salas de rayos $X$ o una sola instalación, se puede definir un "valor típico" como la mediana de la distribución de las dosis medidas y usarse de manera similar. En el caso de DRLs nacionales debería utilizarse una amplia muestra de centros sanitarios y de procedimientos médicos dentales realizados con radiaciones ionizantes en ese país. Finalmente, los DRLs regionales se refieren a grupos de países en los que se hace uso de prácticas similares (International Commission on Radiation Protection, 2017).

Tabla I. Los símbolos de la Comisión Internacional de Unidades y Medidas Radiológicas (ICRU) para las magnitudes de los Niveles de Referencia para Diagnóstico.

\begin{tabular}{lll}
\hline Simbología & Significado & Otros símbolos comunes \\
\hline CTDl ${ }_{\text {vol }}$ & Índice de dosis en tomografía computarizada (volumen) & \\
DLP & Producto dosis longitud & \\
$\mathrm{K}_{\mathrm{a}, \mathrm{i}}$ & Kerma incidente en aire & IAK \\
$\mathrm{K}_{\mathrm{a}, \mathrm{e}}$ & Kerma en aire en la superficie de entrada & ESAK, ESD \\
$\mathrm{P}_{\mathrm{KA}}$ & Producto kerma en aire área & KAP, DAP \\
\hline
\end{tabular}

Esta publicación utiliza la simbología de la ICRU. Se muestran otros símbolos comunes para la conveniencia del lector (Información extraída y adaptada por los autores desde la tabla 2.3 de la publicación ICRP-135 (International Commission on Radiation Protection, 2017)). 
Instalaciones. Para establecer los DRLs, se debe delimitar la zona geográfica en la cual estos serán evaluados y luego aplicados. Un DRL local se puede obtener a partir de un grupo (ej. 10-20) de salas de rayos $X$ o centros sanitarios. En un país desarrollado, con cientos de servicios sanitarios, un muestreo muy detallado representaría una tarea muy complicada, sin embargo, una selección aleatoria de una pequeña proporción de esos centros sanitarios puede ser un buen punto de comienzo. Los resultados de 20-30 servicios pueden ser suficientes en una primera instancia, sólo si se incluye un número suficiente de pacientes. En un país pequeño, con menos de 50 servicios, un muestreo inicial de $30-50 \%$ de estos centros, podría bastar. En estudios posteriores, conforme mejore la infraestructura para la recopilación de datos, el número de servicios incluidos se puede extender para tener una cobertura más representativa (International Commission on Radiation Protection, 2017).

Una vez que se han establecido los DRLs, pueden plantearse estudios de actualización con un intervalo adecuado de 3 a 5 años, pero esto dependerá de las condiciones de cada país o región, considerando el grado de variabilidad de los resultados de los estudios, la introducción de nuevas tecnologías o software de tratamiento de imágenes, y la disponibilidad del personal y recursos para realizar estos estudios (International Commission on Radiation Protection, 2007).

Pacientes o fantomas (maniquíes). La Tabla II de la publicación № 135 de la ICRP (International Commission on Radiation Protection, 2017), describe claramente cuál es el método de evaluación para establecer los DRLs en cada procedimiento de radiología. La gran mayoría de procedimientos basa su estrategia en las medidas realizadas sobre pacientes individuales y clasificadas por edad, peso o ambos. Sin embargo, existen algunas circunstancias limitadas, en las cuales el rendimiento del equipo (output measurement) puede ser evaluado bajo las condiciones estándar para estimar la magnitud correcta. Ejem- plo de lo anterior, sería para el caso de la radiografía intraoral. La Tabla III presenta un resumen del método de establecimiento de DRL para cada tipo de procedimiento radiológico dental.

Es recomendado para los procedimientos radiológicos dentales, donde su método de evaluación sea con pacientes, que al menos se recolecte la información de 20 exámenes (National Radiological Protection Board, 1992). Sin embargo, cuando exista una gran variación o dispersión de los datos, será necesario recolectar información de más procedimientos.

\section{a. Métodos de recolección de datos.}

La recolección manual sigue siendo una opción utilizada, junto con lo anterior, si son pocas las instalaciones, se puede emplear formularios impresos adaptados al examen. Sin embargo, la llegada de los sistemas de información hospitalaria (HIS) y de los sistemas de información radiológica (RIS) permite hacer una revisión retrospectiva de la información de los exámenes de los pacientes. La recolección de datos desde el RIS, permite incluir un número mucho mayor de pacientes. El estándar DICOM, de "Digital Imaging and Communication in Medicine", ha creado un formato específico para estos fines, denominado informe estructurado de dosis de radiación (RDSR) para el registro y almacenamiento de la información de dosis de radiación en las diferentes modalidades de imagen (Digital Imaging and Communications in Medicine, 2007). El sistema de gestión de dosis de pacientes, permite usar la compilación de información de los RDSRs para notificar al personal clínico y a los físicos médicos, cuando las magnitudes dosimétricas excedan algunos de los niveles preestablecidos, especialmente cuando se pueda referir a dosis altas en la piel, existe una alerta para el seguimiento clínico de posibles lesiones por radiación. No obstante, se necesita configurar el envío de los archivos RDSR desde las modalidades al RIS/PACS, así como un software específico que pueda gestionar esta información.

Tabla III. Métodos de evaluación propuestos para establecer niveles de referencia para diagnóstico para cada modalidad de radiología dental.

\begin{tabular}{ll}
\hline Modalidad & Método de evaluación \\
\hline Radiografía intraoral & Medición del rendimiento del equipo en configuración estándar \\
Radiografía panorámica & Medición del PKA O DLP en configuración estándar \\
Radiografía lateral de cráneo & Evaluación en paciente \\
CBCT & Evaluación en paciente \\
\hline
\end{tabular}

CBCT, tomografía computada de haz cónico; PKA, producto kerma en aire área; DLP, producto dosis longitud. (Información extraída y adaptada por los autores desde la tabla 2.1 de la publicación ICRP-135 (International Commission on Radiation Protection, 2017)). 


\section{DISCUSIÓN}

Dado que para los procedimientos radiológicos dentales se utilizan ajustes diferentes, dependiendo si los pacientes son niños o adultos, el establecimiento de los DRL debiera considerar también por separado a cada grupo etario.

En los procedimientos radiológicos (incluida la radiografía intraoral y lateral de cráneo), se establecen los DRL en base a la magnitud kerma en aire en la superficie de entrada $(\mathrm{Ka}, \mathrm{e})$, definida como $\mathrm{Ka}, \mathrm{e}=$ kerma incidente en aire $(\mathrm{Ka}, \mathrm{i})$ x factor de retrodispersión (B), donde $B$ es la radiación retrodispersada que depende de la calidad del haz de radiación y del tamaño del campo. Debido al pequeño tamaño del campo de radiación, los valores de $\mathrm{B}$ en el examen de radiología intraoral son muy pequeños, por lo tanto, muchos autores sugirieron que los valores de $\mathrm{Ka}$,i son casi iguales a los de Ka,e (Kim et al., 2012; Christofides et al., 2016).

Un método conveniente para establecer valores de DRLs y evaluar la dosis en pacientes para radiografía dental es hacer mediciones en configuración estándar. Las unidades intraorales con frecuencia tienen tubos con valores de kilovoltaje y miliampere fijos, y la exposición varía al ajustar el tiempo de exposición según el tipo de pieza dental estudiada. El tiempo de exposición se elige manualmente ya sea con un dial calibrado para la pieza dental o al seleccionar el tiempo de exposición. Las mediciones de Ka,i se pueden hacer en ajustes estándar con un detector de radiación calibrado adecuadamente puesto al final del cono espaciador del equipo de rayos X (Gulson et al., 2007). Por lo general, el equipo para radiografías intraorales se dejará configurado a la sensibilidad de la película o al ajuste de la velocidad del detector digital utilizada en el centro dental. Sin embargo, cuando se realicen las mediciones del rendimiento del equipo o directamente del Ka,i deben asegurarse de que el odontólogo confirme que los ajustes sean los mismos que se utilizaron antes de la medición.

Los estudios de clínicas dentales muestran un rango amplio de niveles de dosis debido a que muchos dentistas han conservado los tiempos de exposición al cambiar a un sistema de película más veloz o instalando un equipo de radiografía digital, en lugar de emplear tiempos de exposición más cortos, que podrían ser apropiados para los receptores de imagen de radiografía digital más sensibles (Gulson et al.; Holroyd, 2012a; Farris \& Spelic, 2015). Las dosis de radiación para ra- diografías intraorales están determinadas por los ajustes de la máquina de rayos $X$, seleccionados en base al tipo de diente, vinculado al tiempo de exposición.

Para radiografías dentales panorámicas, se requieren técnicas que midan las magnitudes $D R L$ de todo el haz. El PKA se puede medir con una cámara de ionización que va conectada a la caja del tubo de rayos $\mathrm{X}$ e intercepta todo el haz, como en radiografías estándar. Como alternativa, se pueden utilizar detectores más pequeños (pero aun así más grandes que el haz de rayos $X$ ), calibrados en condiciones del DLP y posicionados en la ranura de recepción (Holroyd, 2012b; Mitchell \& Martin, 2013).

Por lo general, la CT por haz cónico (CBCT) incluye sistemas de CT por haz cónico dentales y maxilofaciales, haz cónicos utilizados como modalidad de imágenes en fluoroscopios, y sistemas de verificación en radioterapia. Los procedimientos dentales y maxilofaciales están diseñados para visualizar objetos de alto contraste (huesos y aire) con baja exposición a la radiación comparada con la CT convencional, en las que las aplicaciones de fluoroscopía y radioterapia requieren de visualización de estructuras de tejidos blandos y exposiciones que sean sustancialmente más altas, que son comparables a las CT convencionales.

La ICRP recomienda el uso de PKA, Ka,r, CTDIvol, y DLP como magnitudes DRL, dependiendo de la disponibilidad (Tabla II). Tanto PKA como Ka,r tienden a estar disponibles y ser de utilidad para los sistemas de fluoroscopía y CT por haz cónico dental (HPA, 2010), mientras que el CTDIvol y DLP se utilizan para los sistemas de radioterapia por imágenes y algunos sistemas de CT por haz cónico.

Desde 2017, se han hecho pocos avances en el establecimiento de DRLs para CT de haz cónico. Basado en una auditoría preliminar de valores PKA en 41 unidades de CT por haz cónico dentales y maxilofaciales, HPA (2010) propuso un DRL provisional (aunque fue denominado como "dosis alcanzable") de $250 \mathrm{mGy} \cdot \mathrm{cm}^{2}$, normalizado a un área correspondiente a $4,4 \mathrm{~cm}^{2}$ en el isocentro, para la colocación de un primer implante molar en un paciente adulto estándar. Este valor fue adoptado por el consorcio SEDENTEXCT (European Comission, 2012), con la aclaración de que "se necesita trabajo adicional que involucre auditorías a gran escala para establecer DRLs consistentes" para varias aplicaciones de CT por haz cónico dental y maxilofacial. Esta aclaración tam- 
bién es relevante para otras aplicaciones de CT por haz cónico. Los procedimientos de CT por haz cónico dentales y maxilofaciales no deberían exceder la dosis de procedimientos de CT comparables para objetos de alto contraste (típico CTDIvol<10 mGy).

Finalmente, si el análisis revela que se excede constantemente un DRL por cada procedimiento, se debería llevar a cabo una investigación sin retrasos innecesarios, y se deberían efectuar las acciones correctivas apropiadas (European Comission, 2013). Las acciones correctivas (optimización de protección) deberían incluir un análisis del desempeño del equipo, la configuración usada, y los protocolos de examen (Martin, 2011). En general, es más fácil verificar primero la configuración del sistema de rayos $\mathrm{X}$, ya que esto requiere de menos tiempo, luego se analizan los protocolos de examen y finalmente se evalúa cómo es que los operadores aplican los protocolos de examen. Por otro lado, cuando el valor medido de la magnitud del DRL de la instalación es menor que el valor del DRL nacional, la calidad de imagen (o información diagnóstica, cuando se usan múltiples imágenes) debería ser examinada como prioridad en el análisis.

Los odontólogos deberían tener una capacitación sobre radiografías y protección radiológica como parte de su formación (International Commission on Radiation Protection, 2009). Es importante que se mantengan al día y eso incluye información sobre el rol de los DRLs. Esto debería reforzarse a través de retroalimentación en base a los resultados de las mediciones dosimétricas que se hagan. Se recomienda hacer cursos de repaso sobre técnicas radiográficas y de la optimización en protección de radiológica.

Finalmente, se vuelve urgente poder hacer conciencia entre las autoridades sanitarias y políticas en Chile, de incorporar conceptos tales como: control de calidad, programas de garantía de calidad, DRLs, etc., en la normativa nacional, de acuerdo con las últimas recomendaciones del Organismo Internacional de Energía Atómica con el objetivo de hacer más seguro el uso de las radiaciones ionizantes.

\section{AGRADECIMIENTOS}

El investigador Carlos Ubeda agradece el apoyo de la Dirección de Investigación de la Universidad de Tarapacá, a través del proyecto de investigación UTA Mayor N ${ }^{\circ} 7713-18$.
UBEDA, C.; NOCETTI, D.; ARAGÓN, M.; ARAGÓN, G.; ARAGÓN, D. \& MEDINA, O. Diagnostic reference levels in dental radiological procedures: A practical guide. Int. J. Odontostomat., 14(4):610-616, 2020.

SUMMARY: Currently exposure to ionizing radiation is currently the main source of exposure to artificial radiation worldwide, with dental examinations being one of the most frequent events. In order to prevent exposure of unnecessarily high doses in patients, the International Commission on Radiological Protection (ICRP) recommends the use of Diagnostic Reference Levels (DRLs), as an effective tool to help maximize protection from radiation during medical exposure of patients. Given that the Chilean legislation has not yet incorporated the use of DRLs, the purpose of this study was to analyze publication No. 135 of the ICRP to generate a document, to serve as a guide for dentists, didactically outlining in simple but technical language, the main aspects to consider when establishing DRLs in dental radiology procedures.

KEY WORDS: diagnostic reference levels, dental radiology, safety and radiological protection medical exposure.

\section{REFERENCIAS BIBLIOGRÁFICAS}

Christofides, S.; Pitri, E.; Lampaskis, M. \& Papaefstathiou, C. Local diagnostic reference levels for intraoral dental radiography in the public hospitals of Cyprus. Phys. Med., 32(11):1437-43, 2016.

Decreto Supremo No 3 (DS 3). Reglamento de Proteccion Radiologica de Instalaciones Radiactivas. Santiago de Chile, Gobierno de Chile, 1985.

Decreto Supremo No 133 (DS 133). Reglamento sobre Autorizaciones para Instalaciones Radioactivas o Equipos Generadores de Radiaciones lonizantes, Personal que se Desempeña en Ellas, u Opere tales Equipos y Otras Actividades Afines. Santiago de Chile, Gobierno de Chile, 1984.

Digital Imaging and Communications in Medicine (DICOM). Supplement 127: CT Radiation Dose Reporting (Dose SR). DICOM Standards Committee, 2007.

European Comission (EC). Radiation Protection No. 172. Cone Beam CT for Dental and Maxillofacial Radiology - Evidence Based Guidelines. Luxemburgo, European Commission, 2012. Disponible en: http://www.sedentexct.eu/content/ guidelines-cbct-dental-and-maxillofacial-radiology

European Comission (EC). Council Directive 2013/59/Euratom of 5 December 2013 laying down basic safety standards for protection against the dangers arising from exposure to ionising radiation, and repealing Directives 89/618/Euratom, 90/641/Euratom, 96/29/Euratom, 97/43/. Luxemburgo, European Commission, 2013.

Farris, K. \& Spelic, D. Nationwide Evaluation of X-Ray Trends: Highlights of the 2014-15 NEXT Dental Survey. In: Proceedings of 47th National Conference on Radiation Control. CRCPD Publication E-15-4, Frankfort (KY, USA), Conference of Radiation Control Program Directors (CRCPD), 2015. 
Gulson, A. D.; Knapp, T. A. \& Ramsden, P. G. Doses to patients arising from dental xray examinations in the UK 2002-2004. A Review of X-ray protection service data. HPARPD-022. Chilton, Health Protection Agency, Centre for Radiation, Chemical and Environmental Hazards, Radiation Protection Division, 2007.

Hart, D.; Hillier, M. C. \& Wall, B. F. National reference doses for common radiographic, fluoroscopic and dental X-ray examinations in the UK. Br. J. Radiol., 82(973):1-12, 2009.

Hart, D.; Hillier, M. C. \& Shrimpton, P. C. Doses to Patients from Radiographic and Fluoroscopic X-Ray Imaging Procedures in the UK?: 2010 review. Chilton, Health Protection Agency, Centre for Radiation, Chemical and Environmental Hazards, Radiation Protection Division, 2012.

Holroyd, J. R. Trends in Dental Radiography Equipment and Patient Dose in the UK and Ireland. HPA-CRCE-043. Chilton, Health Protection Agency, Centre for Radiation, Chemical and Environmental Hazards, Radiation Protection Division, 2012a.

Holroyd, J. R. The Measurement of X-ray Beam Size from Dental Panoramic Radiography Equipment. HPA-CRCE-032. Chilton, Health Protection Agency, Centre for Radiation, Chemical and Environmental Hazards, Radiation Protection Division, 2012b.

International Commission on Radiation Protection (ICRP). 1990 Recommendations of the International Commission on Radiological Protection. Publication 60. Ann. ICRP, 21(1-3), 1990.

International Commission on Radiation Protection (ICRP). Radiological Protection and Safety in Medicine. Publication 73. Ann. ICRP, 26(2), 1996.

International Commission on Radiation Protection (ICRP). Radiation and your patient - A Guide for Medical Practitioners. ICRP Supporting Guidance 2. Ann. ICRP, 31(4), 2001.

International Commission on Radiation Protection (ICRP). The 2007 Recommendations of the International Commission on Radiological Protection. Ann. ICRP, 37(2-4), 2007a.

International Commission on Radiation Protection (ICRP). Radiological Protection in Medicine. Ann. ICRP, 37(6), 2007b.

International Commission on Radiological Protection (ICRP). Education and training in radiological protection for diagnostic and interventional procedures. Publication 113. Ann. ICRP, 39(5), 2009.

International Commission on Radiation Protection (ICRP). Diagnostic Reference Levels in Medical Imaging. Report 135. Ann. ICRP, 46(1), 2017.

Kim, E. K.; Han, W. J.; Choi, J. W.; Jung, Y. H.; Yoon, S. J. \& Lee, J.S. Diagnostic reference levels in intraoral dental radiography in Korea. Imaging Sci. Dent., 42(4):237-42, 2012.

Martin, C. J. Management of patient dose in radiology in the UK. Radiat. Prot. Dosimetry, 147(3):355-72, 2011.

Martin, C. J.; Le Heron, J.; Borrás, C.; Sookpeng, S. \& Ramirez, G. Approaches to aspects of optimisation of protection in diagnostic radiology in six continents. J. Radiol. Prot., 33(4):711-34, 2013.

Mitchell, S. A. \& Martin, C. J. Comparison of ionisation chamber and semiconductor detector devices for measurement of the dose-width product for panoramic dental units. J. Radiol. Prot., 33(2):321-8, 2013

National Radiological Protection Board (NRPB). National Protocol for Patient Dose Measurements in Diagnostic Radiology. Chilton, Institute of Physical Sciences in Medicine, 1992.

Organismo Internacional de Energía Atómica (OIEA). Protección radiológica y seguridad de las fuentes de radiación: normas básicas internacionales de seguridad. Viena, Normas de seguridad del OIEA para la Protección de las Personas y el Medio Ambiente, Organismo Internacional de Energía Atómica, 2016. Disponible en: https://www-pub.iaea.org/MTCD/ Publications/PDF/P1578_S_web.pdf

Organización Mundial de la Salud (OMS). Comunicando los Riesgos de la Radiación en Radiodiagnóstico Pediátrico. Información para Facilitar la Comunicación sobre los Beneficios y los Riesgos en la Atención Sanitaria. Ginebra, Organización Mundial de la Salud, 2016. Disponible en: https:// apps.who.int/iris/bitstream/handle/10665/272341/ 9789241510349-spa.pdf?ua=1

Ruiz, P. Procedimientos Recomendados para la Dosimetría de Rayos de Energías entre 20 y 150 keV en Radiodiagnóstico. Madrid, Ramírez de Arellano Editores, Sociedad Española de Física Médica, 2005.

United Nations Scientific Committee on the Effects of Atomic Radiations (UNSCEAR). UNSCEAR 2008 Report: Sources and Effects of Ionizing Radiation. Viena, United Nations Scientific Committee on the Effects of Atomic Radiations (UNSCEAR), 2008. Disponible en: http://www.unscear.org/ unscear/publications/2008_1.html

Ubeda, C. Research on safety and radiological protection in dental radiological procedures. How much is there in Chile. Int. J. Odontostomat., 12(2):91-2, 2018.

Ubeda, C.; Nocetti, D. \& Aragón, M. Safety and radiological protection in dental imaging procedures. Int. J. Odontostomat., 12(3):246-51, 2018b.

Direccion para correspondencia:

Carlos Ubeda, PhD.

Departamento de Tecnología Médica

Facultad de Ciencias de la Salud

Universidad de Tarapacá

Arica

CHILE

Email: carlos.ubeda.uta@gmail.com

Recibido : 05-03-2020

Aceptado: 12-05-2020 